\title{
Influence of Female Media Information on Female Media Use Anxiety: Take a Survey of Weibo Users on Social Media as an Example
}

\author{
Mengmeng Guo \\ School of Literature and Journalism, Sichuan University, China \\ \{mengmengguo0726@163.com\}
}

\begin{abstract}
Social media is an important source of influencing women's selfperception and self-awareness. The dimensions of standards and requirements for women contained in women's topic information texts constructed by media will cause women's social comparative behaviors in relevant aspects, and its invisible pressure will lead to anxiety in the use of female media. This research combines quantitative research and qualitative research to study the relationship and main factors of ideal women's anxiety about female media use defined or presented by media. This paper selects the users of Sina Female, an official account with wide influence in the field of female's topics in Sina Weibo, as the interview subjects, and makes qualitative attribution analysis on the influencing factors of female media anxiety according to the grounded theory principle. After that, Structural equations were used to test the effect of variables on female media anxiety. The model includes perceived image information, perceived family and romantic information, perceived occupation stress information, social value, economic value, media stress, and media loyalty. According to the reliability test of the data with a sample size of 318 by SPSS20.0 and AMOS 20.0, the result shows all hypothesis are supported. The study found that perceived external image has the greatest impact on female media use anxiety. Women's perception of economic value is higher than that of social value, and women's anxiety about media use will also produce media loyalty.
\end{abstract}

Keywords: Media Use Anxiety; Social Media; Female Media Image

\section{Introduction}

Wilbur Schramm once pointed out: "Once the medium emerges, it participates in all significant social changes." [1] Undoubtedly, mass media not only delivers information about social events and social changes to the audience, but more importantly, it provides the audience with social role models, popular values, and lifestyles. Diana Crane, an American media culture researcher, explicitly linked the influence of media culture with gender, and found that media culture instilled in the public such cognition as "what does it mean to be a model of a man or a woman" and "from newspapers to music TV, media shapes our 
understanding of gender. By showing us images of women, men and their relationships, the media suggests that we, as men and women, how should our individual images be?" [2]

Mass media is an important source that affects women's self-perception and self-wareness. The ideal women defined or presented by the media are easy to cause women's upward comparison and anxiety to themselves. We speculate that the prevalence of visual culture, the suggestive guidance of media, the lack of feminist consciousness, group pressure and other influencing factors will all become the sources of female media anxiety.

Psychologists have defined the concept of anxiety from different angles. Bekker pointed out that anxiety is a phenomenon in which people feel nervous and vexed in an uncertain environment, while the autonomic nervous system triggers a series of coping behaviors to cope with potential threats[3]. Specific to the relationship between social media and users' anxiety, social media users' anxiety is mainly manifested in the physiological and psychological tension and pressure that users generate when using media. Labrague studies showed that time spent on social networking sites was significantly positively correlated with negative emotions such as anxiety, stress, and depression[4]. Spielberger divides anxiety into two types: state-anxiety and trait-anxiety[5]. Mccord found that users with high social anxiety traits are more likely to experience anxiety when using Facebook than users with low social anxiety traits[6].

Social media anxiety is not only derived from the external characteristics of social media platforms, such as system characteristics and information characteristics, but also influenced by users' personality traits and social communication factors. For example, from the perspective of information, the self-media nature of social media will aggravate users' information compulsion. Faced with such an information environment, users will inevitably suffer from cognitive information barriers when receiving information, resulting in physical and psychological pressure and anxiety[7]. As a form of anxiety, Fear of Missing Out (FoMO) is a phenomenon that has attracted much attention in academia. Cognitive psychologist A. K. Przybylski defined FoMO as "a widespread anxiety that occurs when individuals fail to get the experience they want to know in the event of their absence, mainly manifested as the desire to know what others are doing continuously"[8]. At the same time, in terms of social interaction and personal characteristics of users, some scholars have analyzed the anxiety of social media users. Research in emotional psychology has found that when users' expectations are not met, negative emotions are generally aroused. For example, Osatuyi explored the relationship between personality traits and SNS users' anxiety and privacy concerns and found that creative, neurotic, and conscientious personalities positively affected users' anxiety[9].

According to the current literature, there have been relatively deep researches on the definition, classification and causes of user anxiety, but scholars still lack in-depth and detailed research on the phenomenon of user anxiety in the special context of social media, especially lack of special consideration on the profound impact of social media on women. Relevant literature points out that women are more vulnerable to social media because of their gender characteristics, group pressure, male scrutiny, social prejudice and other factors. At present, there are few researches that comprehensively and comprehensively investigate the multi-dimensional impact of social media on women's anxiety. The anxiety of women's media use needs further investigation and explanation.

\section{Method}

This paper selects the users of Sina Female as the interview subjects, and makes qualitative 
attribution analysis on the influencing factors of female media anxiety according to the grounded theory principle. After that, Structural equations were used to test the effect of variables on female media anxiety. A data with a sample size of 325 will be tested by SPSS20.0 and AMOS 20.0. At present, there is no analysis on the related impact of female media use anxiety in China, and due to the large difference in media use between domestic and foreign users, direct design of non-differentiated structural scales for empirical analysis may not yield reasonable results. Therefore, the qualitative data of this article comes from the in-depth interview data of the users, and adopts the grounded theory in qualitative research to construct the influencing factor model suitable for Chinese women's media use anxiety. The sample of this study comes from female users of weibo, a Chinese social media with one of the largest user groups in the world. Finally, 129 valid samples were obtained. The interview will start from January 6, 2019 to May 12, 2019. After the interview, import the interview content into Nvivo11 software for processing.

Firstly, the researchers scattered and deconstructed the original data through open coding, then identified the views and words with the smallest unit meaning, and named them according to the existing dimensions and text contents of literature research. 54 initial concepts are obtained by acquiring words and expressions with frequency more than 2 times, and 22 categories such as body image, Makeup and hairstyle, comparison psychology and consumption temptation are formed by gathering relevant meanings and themes. On this basis, Axial coding refers to the re-integration of decomposed data to link various categories to achieve a higher level of abstraction. Through the logical connection and combination of the results obtained from the open coding, seven main categories are formed: external image, occupational stress, marriage and family, social value perception, economic value perception, anxiety characterized and subsequent behavior intention. Through the continuous in-depth analysis of the main category, connect it with "storyline", present all the related conditions and behavior phenomena described, and facilitate the formation of the theoretical model. The typical relationship structure of the main category is shown in Table 1.

Table 1. Typical Relationship Structure of the Main Category

\begin{tabular}{|c|c|c|}
\hline Typical relationship & Relationship Structure & Relationship Structure \\
\hline Image $\rightarrow$ Social value & Casual & $\begin{array}{l}\text { Emphasis on appearance leads women's } \\
\text { rights appeal }\end{array}$ \\
\hline Image $\rightarrow$ Economy value & Casual & $\begin{array}{l}\text { Emphasis on appearance leads Women's } \\
\text { Rights Appeal }\end{array}$ \\
\hline $\begin{array}{l}\text { Family \& romantic } \rightarrow \\
\text { Social value }\end{array}$ & Casual & $\begin{array}{l}\text { Agenda setting of family issues leads } \\
\text { jealousy }\end{array}$ \\
\hline $\begin{array}{l}\text { Family \& romantic } \rightarrow \\
\text { Economy value }\end{array}$ & Casual & Agenda setting of family issues leads \\
\hline Social value $\rightarrow$ Stress & Casual & $\begin{array}{l}\text { The social value such as the jealousy } \\
\text { leads anxiety }\end{array}$ \\
\hline Economy value $\rightarrow$ Stress & Casual & The value of consumerism leads anxiety \\
\hline $\begin{array}{l}\text { Occupational Stress } \rightarrow \\
\text { Social value }\end{array}$ & Casual & Occupation stress leads the jealousy \\
\hline $\begin{array}{l}\text { Occupational Stress } \rightarrow \\
\text { Economy value }\end{array}$ & Casual & $\begin{array}{l}\text { Occupation stress leads the hence of } \\
\text { perceived economy risk }\end{array}$ \\
\hline Stress $\rightarrow$ Follow-up behavior & Casual & Anxiety leads blind conformity \\
\hline
\end{tabular}

Based on the typical relationship structure obtained by selective coding, the conceptual model diagram of female media anxiety is constructed by using the relationship path of "information perception, attitude, anxiety emotion, and behavioral intention". The reserved 15 
interview data were selected for saturation test, and the three steps of open coding, spindle coding, and selective coding were repeated, and the comparison and analysis with the existing model showed that other concepts and categories neglected were not found, so the model could be considered as theoretical saturation.

Based on the above conceptual logic relations, summarize the concept of each indicator or select the category concept with the highest frequency in each main category to form the hypothesis of the equation model in this paper. The variable hypothesis includes:

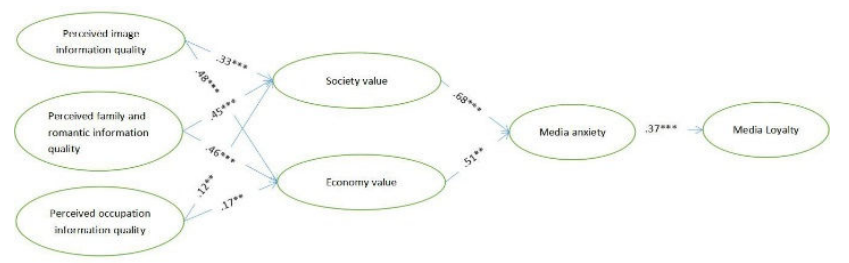

Fig. 1. Model and Hypothesis

Perceived image information quality. Tiggemann, Marika, Miller, Jessica shows internet appearance exposure and magazine reading, but not television exposure, were found to be correlated with greater internalization of thin ideals, appearance comparison, weight dissatisfaction, and drive for thinness[10]. Zhao Xin Rui researched Weibo users, and the hypothesis that image has a significant impact on perceived usefulness was supported[11]. Based on this, the following hypothesis is proposed: H1 perceived image information quality has a positive impact on social value perception; $H 2$ perceived image information quality has a positive impact on economic value perception

Perceive the quality of family and romantic information. At present, there is no professional research on the quality of love and marriage information. However, Wang Zuzhu subdivide the service quality of virtual communities, pointing out that information quality perception of professional virtual communities has a positive impact on users' perceived usefulness and perceived ease of use [12]. Based on this, the research proposes the following hypothesis: $H 3$ perceived that the quality of love and marriage information had a positive impact on social value perception; $H 4$ perceived that the quality of marriage and love information had a positive impact on the economic value perception

Perceived the quality of Occupational stress information. Too many studies have confirmed the positive correlation between occupational stress and economy or social status anxiety, and most researches in this field in China focus on teachers, police, and medical staff [13]. Based on this, the following hypothesis is proposed: H5 Perceived the quality of Occupational stress information has a positive effect on social value perception; H6 Perceived the quality of Occupational stress information has a positive effect on economic value perception;

Social and economic value perception. In terms of social values, Liu Boya believes that there is a significant positive correlation between female secondary school students' negative emotions and social support. In terms of economic value[14], Guo Dexia studied the university teacher's Existential anxiety from the perspective of capital and concluded that increasing economic capital could reduce Existential anxiety, while social capital has little effect[15]. Wang Haoyu pointed out that people troubled by financial problems will have more anxiety, which will lead to insomnia, headache, depression, and other symptoms, affecting individual physical and mental health[16]. Based on these, the research proposes the following hypothesis: $H 7$ social value perception has a positive impact on anxiety; H8 
economic value perception has a positive impact on anxiety

Anxiety. Anxiety is a negative emotion. Anxiety itself is a normal response to stress. In fact, in the field of information systems, there is no uniform conclusion about the effect of anxiety on technology adoption and continuous use. When the newly adopted or introduced technology causes frustration and tension due to difficulties and incompetence, the above situation will occur to individuals. This can lead to a decrease in technology use or an increase in anxiety when using technology: $H 9$ anxiety has a positive impact on Weibo usage loyalty.

\section{Result and Discussion}

\section{The Result of Model Test}

The samples were collected from registered users of Sina Female (7.9 million followers, the most popular female topic weibo in China). 318 valid samples were collected through online and offline questionnaires. According to the reliability test of the data by SPSS20.0, the questionnaire has good reliability. KMO and Bartlett test of spericity were carried out on the data. The KMO test coefficient of sample adequacy was 0.923 , greater than 0.7 . The approximate chi-square value of the Bartlett sphericity test on the samples was 10863.812 , the degree of freedom was 351, and the significance was 0. Principal component analysis (PCA) was used to conduct exploratory factor analysis of the data, and the coefficient whose absolute value was less than 0.5 was removed. The seven common factors effectively explained the overall questionnaire, and the interpretation rate reached $84.583 \%$, which was consistent with the preset latent variables. Then, the Composite Reliability (CR) of the latent variables is tested, and the Composite Reliability of all variables is greater than 0.7 , indicating that the observed variables have a good internal consistency. Besides, the Discriminant Validity of data is tested. Through detection and analysis, it can be found that the AVE values of all variables are greater than 0.6 , indicating that the data has good discrete validity.

AMOS 20.0 was used to set, estimate, and evaluate the model proposed in this paper. The maximum likelihood method was adopted for model parameter estimation to obtain the parameter estimation results of the model and the standardized path coefficient. The t-test method was used to test the significance of the path coefficient, and the results showed that the hypothesis relations were all valid, among which the social value perception had a positive impact on anxiety at the set level of 0.01 , and other hypothesis relations had a positive impact at the set level of 0.001 . The overall fitting degree of the model is tested, and the following fitting indexes are mainly observed, including Absolute Fit Index, Parsimony-Adjusted Index and Relative Fit Index.

Table 2. Model Fit Index

\begin{tabular}{lccccccc}
\hline \multicolumn{1}{c}{ Model Fit } & \multicolumn{8}{c}{ Abdolute Infldex Fit } & \multicolumn{2}{c}{ Parsimony } & Adjusted Index & \multicolumn{2}{c}{ Relative Fix Index } \\
\hline Index & $\mathrm{X}^{2} / \mathrm{df}$ & GFI & RM SEA & PNFI & PGFI & NFI & CFI \\
Reference value & $<3$ & $>0.9$ & $<0.6$ & $>0.5$ & $>0.5$ & $>0.9$ & $>0.9$ \\
Current model & 3.018 & 0.904 & 0.089 & 0.809 & 0.645 & 0.919 & 0.942 \\
\hline
\end{tabular}




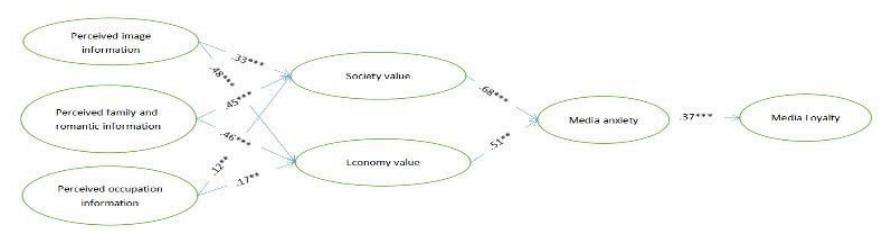

Fig. 2. The Path Effect of the Model

\begin{tabular}{lccccccccc}
\multicolumn{10}{c}{ Table 3. The Result of the Hypothesis } \\
\hline Number & HI & H2 & H3 & H4 & H5 & H6 & H7 & H8 & H9 \\
\hline $\mathrm{P}$ & $<0.001$ & $<0.001$ & $<0.001$ & $<0.001$ & $0.013^{* *}$ & $0.005^{* *}$ & $<0.001$ & $<0.001$ & $<0.001$ \\
& $* * *$ & $* * *$ & $* * *$ & $* * *$ & & & $* * *$ & $* * *$ & $* * *$ \\
Beta $(\beta)$ & 0.33 & 0.48 & 0.45 & 0.46 & 0.12 & 0.17 & 0.68 & 0.51 & 0.37 \\
Result & Support & Support & Support & Support & Support & Support & Support & Support & Support \\
\hline
\end{tabular}

Perceived External Image Information and Female Media Use Anxiety. The total effet shows that perceived external image has the greatest influence on media anxiety. This means that the anxiety of women's media use comes to a large extent from their attention and care for the external image topics such as body, makeup and hairstyle. The commercial "body industry" and "beauty industry" created by social media are important reasons for this phenomenon. In the context of modernity, appearance image has been regarded as a plan that can be processed and completed in this era. The body owner can control and shape the size, shape and appearance of the body by her own will. Due to social media weakens the authority of traditional social norms and social culture in defining the body in some aspects, women are confused and anxious about the concept of a good "female image", while social media seems to provide exactly the value to guide our body. When women carry out specific standards on body aesthetics, the images of women spread by social media will be taken as references by them intentionally or unintentionally. Just like Jacques Lacan's "Mirror image theory", babies confirm their image through the images in the mirror. Jacques Lacan's "mirror image" is, to a certain extent, a symbol that lies outside the main body while positioning the main body. This symbol sets the "ideal self" and pushes us to construct ourselves according to this "ideal self". This "ideal self" is not determined by oneself, and there is a deeper "the other" behind its setting. Social media and the commercial factors contained therein are the "other". The information conveyed to the public by the ideal female image is the presentation of a symbolized female characteristic. In essence, it is an imaginary community, an illusory female body illusion widely existing in society, which expresses what the female body should look like and what form of female body is the beauty these contents. Due to the penetration of commercial factors, the media will intentionally provide a large number of young and beautiful images to depict a fantasy world for women. It is a typical visual myth and an aesthetic fantasy. Under the influence of the body image provided by the media, women's bodies will always be in a state of existential anxiety. Due to the sense of expulsion and uneasiness of anxiety, they will do everything possible to try new methods at any time, catch up with the trend of new fashions and new desires, and pursue certain "cultural ideal exaggeration" in size.

Economic value perception, social value perception, and female media use anxiety. The study found that both economic value perception and social value perception are important factors that lead to women's anxiety in media use, and the impact of economic value perception is higher than that of social value perception. The reason may be related to women's lower economic security and their weaker ability to resist risks to the economy. 
Chinese women face general value confusion. In the workplace, family and individual, women are often in a state of "being torn apart", and their sense of identity in the workplace is insufficient and they are facing multiple pressures.

Female Media Use Anxiety and Media Loyalty. Previous researchers have focused on the fact that the happiness brought by social media to users will produce user stickiness, which will lead to the continuous use of media. However, this study found that the anxiety of media use will also produce the continuous use of media. The reason is firstly related to the dependency of media use. This dependence is an important driving force for users' information behavior, which is reflected in the users' immersion in social software and virtual space. It has the function of cognitive and emotional reinforcement, and will lead to the users' repetition in specific actions, such as frequent and repeated login to social media and continuous addiction to checking microblog status. For example, fear of missing out (FoMO) is a common phenomenon. At the same time, in this study, whether the particularity of women's gender characteristics, economic value perception and social value perception will have an impact on their media use behavior remains to be further explored.

\section{Conclusion}

The study found that women's media use anxiety will also affect subsequent media use behavior, and found multiple factors that affect women's media use anxiety. Subsequent research will further explore the adjustment effect of women's occupation and age level.

\section{References}

[1] W. Schramm. Mass Media and National Development. Stanford University Press, 1971.

[2] D. Crane."The Production of Culture: Media and the Urban Arts". SAGE Publication, 1992.

[3] H.L. Bekker, F. Legare, D. Stacey. "Is anxiety a suitable measure of decision aid effectiveness: A systematic review?". Patient Education and Counseling. Vol. 50, No.3.pp. 255-262, 2009.

[4] L.J. Labrague. "Facebook use and states of depression, anxiety and adolescents' emotional stress". Health Science Journal, Vol. 8. No. 1. pp. 80-89,2014.

[5] C.D. Spielberger, L. Gorsuch, L. Lushene. Manual for the state-trait anxiety inventory. Consulting Psychologists Press, 1983.

[6] B. Mccord B, T.L. Odebaugh, C.A. Levinson. "Facebook: Social uses and anxiety". Computers in Human Behavior, Vol. 34. No. 34, pp. 23-27, 2014.

[7] W. Xirong. "A Review of Library Anxiety and Its Research". Journal of Academic Libraries, No. 3, pp. 6-12. 2009.

[8] Przybylskiak, Koum, Dehaancr. "Motivational,emotional,and behavioral correlates of fear of missing out". Computers in human behavior. Vol. 29, No. 4, pp.1841-1848, 2013.

[9] B. Osatuyi."Personality traits and information privacy concern on social media platforms". Journal of Computer Information Systems, Vol. 55. No. 4., pp. 11-19, 2015.

[10] M. Tiggemann, A. Slater, NetTweens. "The Internet and Body Image Concerns in Preteenage Girls". The Journal of Early Adolescence, Vol. 34, No. 5, pp. 606-620, 
2014.

[11] Z. Xinrui. Research on Users' Intention of Continuous Use of Microblog Based on Network Externality and Social Impact. Harbin: Northeast University of Finance and Economics, 2012.

[12] W. Zuzhu, W. Lei. "Research on Influencing Factors of User Satisfaction with Participation in Professional Virtual Communities". Journal of Wuhan University of Technology, Vol. 10, pp.784-788, 2013.

[13] X. Xiaohong. "Comparison and Empirical Study of Three Surveys on Teachers' Occupational Stress". Shanghai research on education, Vol. 8, No. 1, .pp. 65-69, 2017.

[14] L. Boya. "Study on Negative Emotion, Loneliness and Social Support of Female Nursing Students in Secondary Technical Schools". Sichuan Mental Health, Vol. 6, No. 1, pp.258-261, 2019.

[15] G. Dexia,"Research on University Teachers' Existential Anxiety: A Capital Perspective-Based on Empirical Analysis of Beijing A University Teachers' Group", Research on Teacher Education. Vol. 7, pp. 60-67, 2019.

[16] W. Haoyu, F. Fang."Structure, Measurement and Influencing Factors of Chinese Wealth Anxiety Symptoms", Scientia Sinica, Vol.8, pp. 996-1012, 2019.

[17] Q.J.J. Wong, R.M. Rapee. "The aetiology and maintenance of social anxiety disorder: A synthesis of complimentary theoretical models and formulation of a new integrated model. Journal of Affective Disorders, Vol. 203, pp. 84-100, 2016. 